\title{
TSPO PET Identifies Different Anti-inflammatory Minocycline Treatment Response in Two Rodent Models of Epileptogenesis
}

\author{
Bettina J. Wolf ${ }^{1,2} \cdot$ Mirjam Brackhan ${ }^{1,2,3} \cdot$ Pablo Bascuñana ${ }^{1} \cdot$ Ina Leiter ${ }^{1,2,4} \cdot$ B. Laura N. Langer ${ }^{1} \cdot$ Tobias L. Ross $^{1}$. \\ Jens P. Bankstahl ${ }^{1}$ (D) $\cdot$ Marion Bankstahl ${ }^{2,5}$
}

Published online: 22 January 2020

(C) The Author(s) 2020

\begin{abstract}
Epileptogenesis-associated brain inflammation might be a promising target to prevent or attenuate epileptogenesis. Positron emission tomography (PET) imaging targeting the translocator protein (TSPO) was applied here to quantify effects of different dosing regimens of the anti-inflammatory drug minocycline during the latent phase in two rodent models of epileptogenesis. After induction of epileptogenesis by status epilepticus (SE), rats were treated with minocycline for 7 days ( $25 \mathrm{or} 50 \mathrm{mg} / \mathrm{kg}$ ) and mice for 5 or 10 days ( 50 or $100 \mathrm{mg} / \mathrm{kg}$ ). All animals were subjected to scans at 1 and 2 weeks post-SE. Radiotracer distribution was analyzed and statistical parametric mapping (SPM) was performed, as well as histological analysis of astroglial activation and neuronal cell loss. Atlas-based analysis of $\left[{ }^{18} \mathrm{~F}\right] \mathrm{GE} 180$ PET in rats revealed a dose-dependent regional decrease of TSPO expression at 2 weeks post-SE. Results of SPM analysis depicted a treatment effect already at 1 week post-SE in rats treated with the higher minocycline dose. In mice, TSPO PET imaging did not reveal any treatment effects whereas histology identified only a treatment-related reduction in dispersion of dentate gyrus neurons. TSPO PET served as an auspicious tool for temporal monitoring and quantification of anti-inflammatory effects during epileptogenesis. Importantly, the findings underline the need to applying more than one animal model to avoid missing treatment effects. For future studies, the setup is ready to be applied in combination with seizure monitoring to investigate the relationship between individual early treatment response and disease outcome.
\end{abstract}

Key Words Neuroinflammation $\cdot$ translocator protein $\cdot$ PET $\cdot$ epileptogenesis $\cdot$ minocycline

Bettina J. Wolf and Mirjam Brackhan as well as Jens P. Bankstahl and Marion Bankstahl contributed equally to this work.

Jens P. Bankstahl

bankstahl.jens@mh-hannover.de

1 Department of Nuclear Medicine, Hannover Medical School, Carl-Neuberg-Str. 1, 30625 Hannover, Germany

2 Department of Pharmacology, Toxicology and Pharmacy, University of Veterinary Medicine, Hannover, Germany

3 Present address: Department of Pathology, Section of Neuropathology, University of Oslo and Oslo University Hospital, Oslo, Norway

4 Present address: Institute of Neuroanatomy and Cell Biology, Hannover Medical School, Hannover, Germany

5 Department of Laboratory Animal Science, Hannover Medical School, Hannover, Germany

\section{Introduction}

With 2.4 million people diagnosed with epilepsy worldwide each year, it is one of the most common chronic brain diseases [1]. Despite the availability of numerous anti-epileptic drugs, in approximately $30 \%$ of all epilepsy patients, these medications fail to suppress the spontaneously recurring epileptic seizures [2]. Thus, it is desirable to develop therapeutic strategies that target the mechanisms underlying disease development rather than the symptoms of epilepsy [3]. Temporal lobe epilepsy, the most drug-refractory type of epilepsy [4], is frequently preceded by brain insults, which initiate a cascade of changes in the brain resulting in epilepsy development $[5,6]$. The period between the initiating insult and the first clinically obvious seizures, termed "latent period," offers a window of opportunity for anti-epileptogenic treatment. For evaluation of anti-epileptogenic therapies, post-status epilepticus (SE) models are an accepted and widely used tool [7]. However, no epilepsy-preventive therapeutic strategies have been identified to date [8]. 
Prolonged inflammatory responses of the brain, characterized by sustained microglial and astroglial activation as well as overexpression of pro-inflammatory molecules, have been proposed to play a causative role in epileptogenesis $[9,10]$. Evidence of inflammatory processes were revealed by ex vivo techniques such as immunohistochemistry or autoradiography not only in several post-SE animal models [11-13] but also in human brain specimens from structural forms of epilepsy with an acquired or genetic etiology as recently reviewed [14]. Moreover, the availability of dedicated small animal positron emission tomography (PET) scanners allows serial quantitative in vivo imaging of epileptogenesis-associated inflammatory processes [11], targeting inflammation-related molecules such as the translocator protein (TSPO), which is predominantly expressed by activated microglia $[15,16]$. Recently, our group has elucidated the spatiotemporal profile of microglial activation during epileptogenesis both in the lithium-pilocarpine rat model and in the intrahippocampal kainate mouse model by longitudinal TSPO PET imaging and complementary in vitro techniques $[17,18]$. In these models, microglial activation was evident in epilepsyassociated brain regions such as the hippocampus from 2 days to at least 3 weeks after status epilepticus (SE), reaching its maximum at about 1-2 weeks depending on the brain region. Considering these findings, early anti-inflammatory treatment may represent a promising strategy to counteract epileptogenesis after brain insults [19] and treatment effects may be monitored by in vivo imaging of brain inflammation.

Minocycline represents a promising candidate for antiinflammatory treatment during epileptogenesis $[20,21]$. Despite often referred to as an inhibitor of microglial activation, minocycline affects either directly or indirectly also other cells types such as neurons, astrocytes, and oligodendrocytes. The specific mechanism of its anti-inflammatory activity is unknown. Nevertheless, various preclinical studies have revealed both disease-modifying effects on epileptogenesis and/or effects on neuropathological hallmarks of epilepsy like neurodegeneration or neuroinflammation [22-25]. However, in vivo assessment of minocycline effects on its supposed main target, i.e., activated microglia, cannot be achieved by using only standard tissue extraction-based techniques. Furthermore, assessing the interaction between early treatment effects and later disease outcome in individual animals is not possible by using histology. To facilitate such investigations, studies validating the utility of preclinical TSPO PET as a non-invasive monitoring tool for anti-inflammatory treatment response during the latent phase are needed.

In the present study, we quantified the antiinflammatory efficacy of minocycline treatment during the latent period in the pilocarpine rat model and the intrahippocampal kainate mouse model by serial in vivo TSPO PET. We complemented PET imaging data by in vitro autoradiography using the same radio-ligand as well as histological evaluation of treatment impact on neurodegeneration and astrogliosis.

\section{Materials and Methods}

\section{Animals}

Adult female Sprague-Dawley rats (200-220 g) were purchased from Harlan (Italy). Male NMRI mice were obtained from Charles River Laboratories (Sulzfeld, Germany) at the age of 7 weeks. The choice of sex is based on earlier work of our and the Löscher research group [17, 18, 26, 27]. Despite the use of both sexes is desirable, we decided to stick to the established protocols, resulting in reasonable epileptogenesis induction rates with low mortality, as it has been shown before that the change of sex, strain, or breeder can significantly influence epileptogenesis characteristics [26-28]. Rats were housed in pairs whereas mice were housed in groups under controlled climate and controlled hygienic conditions in individually ventilated cages under a 14/10-h light-dark cycle. They received autoclaved tap water and standard laboratory chow (Altromin 1324, Lage, Germany) ad libitum. Animals were allowed to adapt to the new conditions for at least 1 week and were handled repeatedly before the experiments started. Experiments were formally approved by the responsible local authority and were conducted in accordance with European Communities Council Directives 86/609/EEC and 2010/63/ EU. All efforts were made to minimize pain, suffering, and the number of animals. The principles outlined in the ARRIVE guidelines and the Basel declaration including the $3 \mathrm{R}$ concept have been considered.

\section{General Study Design}

Animals were randomly assigned to experimental groups. Subsequent to SE induction by pilocarpine or intrahippocampal kainate injection, animals underwent either minocycline or vehicle treatment. $\left[{ }^{18} \mathrm{~F}\right] \mathrm{GE} 180 \mathrm{PET} / \mathrm{CT}$ scans were performed 1 and 2 weeks after SE induction. Scan time points were selected based on findings previously reported by our group $[17,18]$. In a recent study, the latency phase in the pilocarpine rat model was found to range from 6 to 10 days post-SE (mean 7 days) [29]. The latency phase for male NMRI mice in the intrahippocampal mouse model was found to be 10-14 days post-SE [26]. Therefore, in average, the first imaging time point lays within the latency phase whereas the second represents the early disease phase. After the second scan, all animals were euthanized for further analysis of brain slices by in vitro autoradiography and immunohistochemistry. Unless stated otherwise, all chemicals used were of analytic grade and purchased from Sigma-Aldrich (Steinheim, Germany). 


\section{Post-SE Models of Epileptogenesis}

SE in rats and mice was induced as described elsewhere $[17,18]$. Shortly, 14-16 h after pretreatment with lithium chloride $(127 \mathrm{mg} / \mathrm{kg}$ p.o.) and $30 \mathrm{~min}$ after pretreatment with methyl-scopolamine $(1 \mathrm{mg} / \mathrm{kg}$ i.p. $)$, rats $(n=34)$ received rationed i.p. injections of pilocarpine (max. $60 \mathrm{mg} / \mathrm{kg}$ ). Behavioral seizures were assessed according to Racine's scale [30]. Convulsive SE was interrupted after $90 \mathrm{~min}$ by diazepam (Ratiopharm $\mathrm{GmbH}$, Ulm, Germany, max. $25 \mathrm{mg} / \mathrm{kg}$ i.p.). Mice $(n=36)$ were anesthetized with choral hydrate $(500 \mathrm{mg} / \mathrm{kg}$, i.p.). Kainate monohydrate $(0.21 \mu \mathrm{g}$ in $50 \mathrm{~nL}$ saline $)$ was injected over $60 \mathrm{~s}$ into the right cornu ammonis subregion 1 (CA1) of the dorsal hippocampus. The average time under chloral hydrate anesthesia was $124 \pm 42 \mathrm{~min}$. Kainate injection took place about $40 \mathrm{~min}$ after begin of anesthesia. Mice were closely observed for at least $2 \mathrm{~h}$ for clinical signs of SE upon awakening from anesthesia and 1 mouse died shortly after SE. Typically, every mouse shows SE symptoms within this period, which was also the case in this study. SE was not interrupted in mice. In the days following SE, animals were closely monitored, received mashed laboratory chow, and were injected with glucose electrolyte solution (Sterofundin HEG-5, Braun) until resuming normal feeding behavior. Animals resumed normal feeding within $2.76 \pm 2.90$ and $3.57 \pm 0.76$ days postSE for mice and rats, respectively.

\section{Minocycline Treatment}

Minocycline (minocycline hydrochloride; Tokyo Chemical Industry) was freshly dissolved in saline and administered i.p. in a volume of $2 \mathrm{ml} / \mathrm{kg}$ in rats and $10 \mathrm{ml} / \mathrm{kg}$ in mice. Rats received either $25(n=7)$ or $50 \mathrm{mg} / \mathrm{kg}(n=5)$ daily for 7 days starting $24 \mathrm{~h}$ post-SE. Mice received either $50 \mathrm{mg} / \mathrm{kg}$ once daily over 5 days $(n=7)$ or $50 \mathrm{mg} / \mathrm{kg}$ twice daily over 10 days $(n=9)$, both starting $6 \mathrm{~h}$ after SE. Vehicle treatment was performed with saline instead of minocycline solution (mice $n=19$; rats $n=9$ ). Dosing regimens were derived from earlier studies in mice and rats. In the pilocarpine-induced SE, rat model reduced microglial activation, reduced production of IL-1 $1 \beta$ and TNF $\alpha$, and prevention of neuronal cell loss was observed after treatment with $45 \mathrm{mg} / \mathrm{kg}$ minocycline once daily for 14 days. Further, attenuation of spontaneous recurrent seizure development indicating disease-modifying properties regarding epilepsy development was described [25]. In mice, a pronounced reduction in microglia activation after systemic kainate-induced SE at postnatal day 25 was mediated by minocycline treatment over 6 days $(20 \mathrm{mg} / \mathrm{kg})$ [22]. The dosing regimens were adapted to stop the treatment before the first PET scan. As the $50 \mathrm{mg} / \mathrm{kg}$ dose resulted in reduced microglia activation in rats, we started with this dose in the mouse model.

\section{PET Imaging}

PET imaging and the synthesis of $\left[{ }^{18} \mathrm{~F}\right] \mathrm{GE} 180$ was performed as described before $[17,18]$ with a resulting specific activity from 444.8 to $588.6 \mathrm{GBq} / \mu \mathrm{mol}$. Due to logistical limitations it was not possible to scan all animals at all time points. Under isoflurane anesthesia, animals were placed prone in an imaging chamber with the brain at the center field of view of a dedicated small animal PET/CT camera (Inveon PET scanner, Siemens). $\left[{ }^{18} \mathrm{~F}\right] \mathrm{GE} 180(23.32 \pm 4.25 \mathrm{MBq}$ in rats, $12.85 \pm$ $0.66 \mathrm{MBq}$ in mice) was injected into a lateral tail vein. In rats, dynamic data were acquired over $1 \mathrm{~h}$ in 32 frames as described before [18]. In mice, a static 20min PET acquisition was performed after a 40-min uptake period under anesthesia, each followed by a lowdose CT scan. For reconstruction of images, an iterative ordered subset expectation maximization three-dimensional/maximum posteriori (OSEM3D/fastMAP) algorithm with standard corrections including transmission correction was applied.

Image analysis and kinetic modeling were completed using PMOD software (PMOD 3.7, Zürich, Switzerland). For coregistering PET images to an MRI template [31, 32], CT images were matched to both the corresponding PET images and the MRI template. Subsequently, a volume of interest (VOI) template based on the MRI atlas was applied to the co-registered images $[33,34] .\left[{ }^{18} \mathrm{~F}\right] \mathrm{GE} 180$ uptake was quantified as percent injected dose per gram tissue $(\% \mathrm{ID} / \mathrm{g})$ in hippocampus, thalamus, and piriform cortex in rats or whole cortex in mice.

For kinetic modeling of PET data gained in rats, VOIs $\left(16.0 \mathrm{~mm}^{3}\right.$, cuboid) were defined for the left and right carotid artery in the dynamic images and averaged in order to generate time activity curves of whole blood [35, 36]. Using these image-derived input functions, time activity curves were fitted to a two-tissue compartment model [37] in order to obtain the volume of distribution $\left(\mathrm{V}_{\mathrm{t}}\right)$. Region-based Logan graphical analysis was additionally performed (data not shown) and both methods provided similar results. Additionally, $\mathrm{V}_{\mathrm{t}}$ maps were calculated in PMOD by voxel-wise modeling using the Logan plot.

Calculation of averaged images and statistical parametric mapping (SPM) was performed using MATLAB software (The MathWorks) and SPM12 (University College London). For SPM analysis, differences between vehicle and minocycline-treated animals were calculated by unpaired 2sample $t$ tests setting a significance level threshold of 0.01 (uncorrected for multiple comparisons). The minimum cluster size of voxels was set to 100 for rats and to 50 for mice. 


\section{Autoradiography and Immunohistology}

After the last PET/CT scan, animals were euthanized, and brains were rapidly removed, covered with Tissue-Tek ${ }^{\circledR}$ (Sakura ${ }^{\circledR}$ Finetek, USA), snap-frozen, and stored at $-20{ }^{\circ} \mathrm{C}$. Coronal $14-\mu \mathrm{m}$-thick slices were cut in a cryostat (Microm HM560; Schwerte, Germany) and mounted on Histobond slides (Marienfeld, Germany). Rat brains were sliced at section levels -3.6 and $-5.2 \mathrm{~mm}$ relative to bregma [38] and mouse brains at -1.82 and $-2.92 \mathrm{~mm}$ relative to bregma [39], in order to include thalamus, hippocampus and (piriform/entorhinal) cortex as regions for tissue analysis. The administration of radiotracers for PET imaging before killing the animals does not interfere with later performed autoradiography or immunohistochemistry, as the used radiotracers are administered only in nano- to picomolar concentrations, and are therefore much below target saturation.

Autoradiography was performed as described earlier [18]. Brain sections were incubated with $\left[{ }^{18} \mathrm{~F}\right] \mathrm{GE} 180(\sim 2 \mathrm{MBq}$ per $100 \mathrm{ml}$ PBS), exposed to a high-resolution phosphor imaging plate (PerkinElmer), and digitized using Cyclone scanner (PerkinElmer). Images were co-registered to the 3D VOI atlas, using PMOD software. To calculate activity concentration $\left(\mathrm{Bq} / \mathrm{mm}^{2}\right)$, gray scale values were interpolated to a calibration curve $(10-500 \mathrm{kBq} / \mathrm{ml})$.

As increased TSPO PET signals were confirmed by immunohistochemistry of activated microglia during epileptogenesis before $[18,40]$, here, we focused on immunohistochemical staining of astrocytes, for which TSPO expression under certain conditions has also been shown [40]. Furthermore, neurodegeneration was investigated as a marker for potential neuroprotective minocycline effects. Brain slices, fixated with $4 \%$ paraformaldehyde, were immunostained for the astrocyte marker glial fibrillary acidic protein (polyclonal rabbit anti-GFAP antibody, Dako), or the neuronal marker neuronal nuclear antigen (NeuN; mice, \#ABN78 Millipore; rats, MAB377) applying a previously described procedures $[18,41]$. Slices were incubated with Vectastain $\mathrm{ABC}$ reagent (Biozol) and subsequently stained by nickel-intensified diaminobenzidine reaction.

The severity of neuronal damage and astrocyte activation was assessed in hippocampus and thalamus in both slicing levels mentioned above and values averaged afterwards. Subregions of the hippocampus were scored separately and values averaged afterwards. For scoring of neurodegeneration in NeuN-stained sections, a semi-quantitative system was adapted from Polascheck et al. [42]: 0, no obvious neuronal cell loss; 1 , $<20 \%$ neuronal cell loss; 2, 20-50\% neuronal cell loss; 3, > 50-75\% neuronal cell loss; $4,>75 \%$ neuronal cell loss. In the same slices, dispersion of the dentate gyrus (DG) neurons was scored: 0 , no dispersion; 1 , slight dispersion, cells are packed less densely; 2, moderate dispersion, gaps between cells; 3 , severe dispersion, big gaps between cells; 4 , very severe dispersion, many cells are separated from each other. Additionally, the number of neurons in the dentate hilus was quantified in rat brain slices using AxioVision software (Zeiss, Jena, Germany) as described before [42]. Astroglia activation score values were defined as: 0 , resting, $<10 \%$ activated cells; 1 , mostly resting, approximately $30 \%$ activated cells; 2 , approximately $60 \%$ activated cells, some resting; $3,>90 \%$ activated cells, densely packed [18]. Every analysis was performed at 100-timesmagnification (Leica, Wezlar, Germany) and investigators were blinded to experimental groups. Slices were chosen randomly. For autoradiography, two (rats) or three (mice) slices per animal and section level were analyzed and the mean was calculated. For histology, one slice per animal, staining and section level was assessed. Mean of left and right hemisphere was calculated (only rats) followed by calculation of the mean of section levels (mice and rats).

\section{Statistical Analysis}

Statistical analyses was performed using Prism7 (GraphPad Software, La Jolla, CA, USA) software. All data are presented as mean \pm standard deviation (SD). Values of $p<0.05$ were considered statistically significant. For data of rats post-SE, one-way analysis of variance (ANOVA) with Dunnett's post hoc test for multiple comparisons was used to test for differences between minocycline- and vehicle-treated rats at the same time point in $\left[{ }^{18} \mathrm{~F}\right] \mathrm{GE} 180 \mathrm{~V}_{\mathrm{t}}$, uptake, and in vitro binding, as well as hilar neuronal density. Non-parametric scoring data of histological analyses were compared using the Kruskal-Wallis test, followed by Dunn's post hoc test for multiple comparisons of minocycline- versus vehicle-treated groups. For data of mice, Student's $t$ test was applied to compare $\left[{ }^{18} \mathrm{~F}\right] \mathrm{GE} 180$ uptake in minocycline- and vehicle-treated groups. Results of histological analyses were assessed using Mann-Whitney test. Correlations of in vivo PET data and in vitro autoradiography were calculated by Pearson's linear regression analysis. Required minimal group sizes for both animal models $(n=6)$ were estimated using power analysis (G*Power, Kiel University, Germany; two-tailed unpaired $t$ test, power: $0.8, \alpha$-error: 0.05 , effect size: 2.0 ). If possible, additional animals were included to account for a certain drop-out rate.

\section{Results}

\section{Lithium-Pilocarpine Post-SE Rat Model}

Thirty out of 34 rats reached SE (overall induction rate $88 \%$ ), requiring an average pilocarpine dose of $35.3 \pm 5.1 \mathrm{mg} / \mathrm{kg}$. Rats which did not develop SE were excluded from all further experiments. Within the first $72 \mathrm{~h}$ after SE 8 animals died (mortality rate $26.7 \%$ ). In addition, one animal of the lowdose minocycline group died 6 days after SE. 
In a previous study in the pilocarpine rat model, we already determined the SE-induced magnitude of TSPO increase in comparison to baseline data by $\left[{ }^{11} \mathrm{C}\right] \mathrm{PK} 11195$ PET [18]. After SE, $\left[{ }^{11} \mathrm{C}\right] \mathrm{PK} 11195$ uptake was elevated in most brain regions, including those associated with epileptogenesis (hippocampus, piriform cortex, thalamus) but not the cerebellum. The increase in TSPO binding reached its peak approximately 7 days after SE (up to 2.08-fold increase in the ventral hippocampus) and remained significantly above baseline values up to 3 weeks after SE.

Effects of minocycline treatment on epileptogenesisassociated microglial activation were evaluated by repeated $\left[{ }^{18} \mathrm{~F}\right] \mathrm{GE} 180$ PET imaging of TSPO expression. Atlas-based $\left[{ }^{18} \mathrm{~F}\right] \mathrm{GE} 180$ uptake analysis did not reveal significant differences between groups, except for a decrease of TSPO expression 2 weeks after SE in the thalamus of rats treated with $50 \mathrm{mg} / \mathrm{kg}$ minocycline in comparison to vehicle-treated rats $(p=0.023$, Table 1). However, we observed significant reduction of TSPO expression in several brain regions when comparing $\left[{ }^{18} \mathrm{~F}\right] \mathrm{GE} 180$ volume of distribution $\left(\mathrm{V}_{\mathrm{t}}\right)$ between vehicleand minocycline-treated rats (Fig. 1a, c). At 1 week post-SE, no changes could be identified in the low-dose $(25 \mathrm{mg} / \mathrm{kg})$ minocycline-treated animals, neither in the atlas-based nor in the SPM analysis. High dose $(50 \mathrm{mg} / \mathrm{kg})$ minocycline-treated rats showed no decrease considering only the atlas-based analysis, but SPM identified decreased voxels in the hippocampus and the thalamus (Fig. 1b). At 2 weeks post-SE, $V_{t}$ was significantly lower for both minocycline doses. In rats treated with $25 \mathrm{mg} / \mathrm{kg}$ minocycline, $\mathrm{V}_{\mathrm{t}}$ was up to $22 \%$ lower in the thalamus than in vehicle-treated rats $(p=0.012) . \mathrm{V}_{\mathrm{t}}$ in rats treated with $50 \mathrm{mg} / \mathrm{kg}$ minocycline was even more decreased, reaching maximal reduction also in the thalamus $(-36 \%, p<0.001)$. SPM confirmed the atlas-based analysis 2 weeks post-SE (Fig. 1b).

In vitro autoradiography of brain slices generated from rats at 14 days after SE induction (Table 1) also revealed decreased $\left[{ }^{18} \mathrm{~F}\right] \mathrm{GE} 180$ in vitro binding in minocycline-treated animals compared to the vehicle-treated group. This reached significance in the thalamus ( $13 \%$ reduction, $p=0.018)$ and the piriform cortex $(15 \%$ reduction; $p=0.001)$ in rats receiving $50 \mathrm{mg} / \mathrm{kg}$ minocycline, although there was a trend for reduced $\left[{ }^{18} \mathrm{~F}\right] \mathrm{GE} 180$ binding in the piriform cortex in rats treated with the lower minocycline dose $(p=0.079)$. To confirm validity of the in vivo data, correlation analysis between in vitro and in vivo data was performed, and $\left[{ }^{18} \mathrm{~F}\right] \mathrm{GE} 180$ binding showed a very strong correlation with both in vivo uptake $(r=0.816$, $p<0.001)$ and $\mathrm{V}_{\mathrm{t}}(r=0.785, p<0.001)$.

Brain slices of rats 2 weeks after SE were immunostained for neurons (NeuN) and astrocytes (GFAP; Fig. 2a, d). Previously published comparisons with naïve rats revealed clear glial activation as well as neurodegeneration in rats 2 weeks post-SE [18]. However, scoring of neuronal cell loss and astrocyte activation did not reveal any treatment effects (Fig. 2b, e). This was also true for the neuronal density in the hippocampal dentate hilus (Fig. 2c).

\section{Intrahippocampal Kainate Post-SE Mouse Model}

All animals undergoing intrahippocampal kainate injections showed typical signs of SE (circling, head tilt, head nodding, chewing, mild convulsions of forelimbs). One minocyclinetreated mouse $(50 \mathrm{mg} / \mathrm{kg}$ once daily) died during PET scanning at 1 week after SE. Mice treated twice daily with $50 \mathrm{mg} / \mathrm{kg}$ minocycline developed severe diarrhea within several days. Therefore, this treatment protocol did not meet the criterion of acceptable tolerability and mice were excluded from all further experiments.

Mice underwent $\left[{ }^{18} \mathrm{~F}\right] \mathrm{GE} 180$ PET scans at 7 and 14 days after SE induction (Fig. 3). In a previous study, at around 1 week after SE, a distinct elevation of TSPO signal became apparent in the ipsilateral dorsal hippocampus (2.17-fold), but also in the ipsilateral ventral hippocampus (1.64-fold), thalamus (1.58-fold), and cortex (1.44-fold). At 14 days post-SE,

Table 1 In vivo brain uptake and in vitro autoradiography of $\left[{ }^{18} \mathrm{~F}\right] \mathrm{GE} 180$ in rats after lithium-pilocarpine-induced SE

\begin{tabular}{|c|c|c|c|c|c|c|c|}
\hline & & \multicolumn{2}{|l|}{ Hippocampus } & \multicolumn{2}{|l|}{ Thalamus } & \multicolumn{2}{|c|}{ Piriform cortex } \\
\hline & & Mean \pm SD & $p$ value & Mean \pm SD & $p$ value & Mean \pm SD & $p$ value \\
\hline \multirow[t]{3}{*}{ Uptake 1 week post-SE [\% ID/g] } & Vehicle & $0.60 \pm 0.08$ & & $0.57 \pm 0.11$ & & $0.60 \pm 0.11$ & \\
\hline & Mino 25 & $0.53 \pm 0.13$ & 0.434 & $0.47 \pm 0.11$ & 0.252 & $0.56 \pm 0.13$ & 0.792 \\
\hline & Mino 50 & $0.52 \pm 0.08$ & 0.328 & $0.44 \pm 0.10$ & 0.132 & $0.57 \pm 0.11$ & 0.814 \\
\hline \multirow[t]{3}{*}{ Uptake 2 weeks post-SE [\%ID/g] } & Vehicle & $0.47 \pm 0.05$ & & $0.46 \pm 0.05$ & & $0.50 \pm 0.03$ & \\
\hline & Mino 25 & $0.43 \pm 0.08$ & 0.398 & $0.40 \pm 0.07$ & 0.192 & $0.46 \pm 0.05$ & 0.328 \\
\hline & Mino 50 & $0.45 \pm 0.04$ & 0.681 & $0.37 \pm 0.03$ & 0.023 & $0.50 \pm 0.08$ & 0.999 \\
\hline \multirow[t]{3}{*}{ In vitro autoradiography 2 weeks post-SE $\left[\mathrm{Bq} / \mathrm{mm}^{2}\right]$} & Vehicle & $42.89 \pm 3.26$ & & $39.06 \pm 1.34$ & & $49.14 \pm 4.86$ & \\
\hline & Mino 25 & $42.46 \pm 1.66$ & 0.955 & $36.80 \pm 2.00$ & 0.733 & $47.66 \pm 3.71$ & 0.079 \\
\hline & Mino 50 & $41.66 \pm 1.57$ & 0.701 & $33.94 \pm 1.31$ & 0.018 & $41.63 \pm 3.00$ & 0.001 \\
\hline
\end{tabular}

SE, status epilepticus; Mino, minocycline 


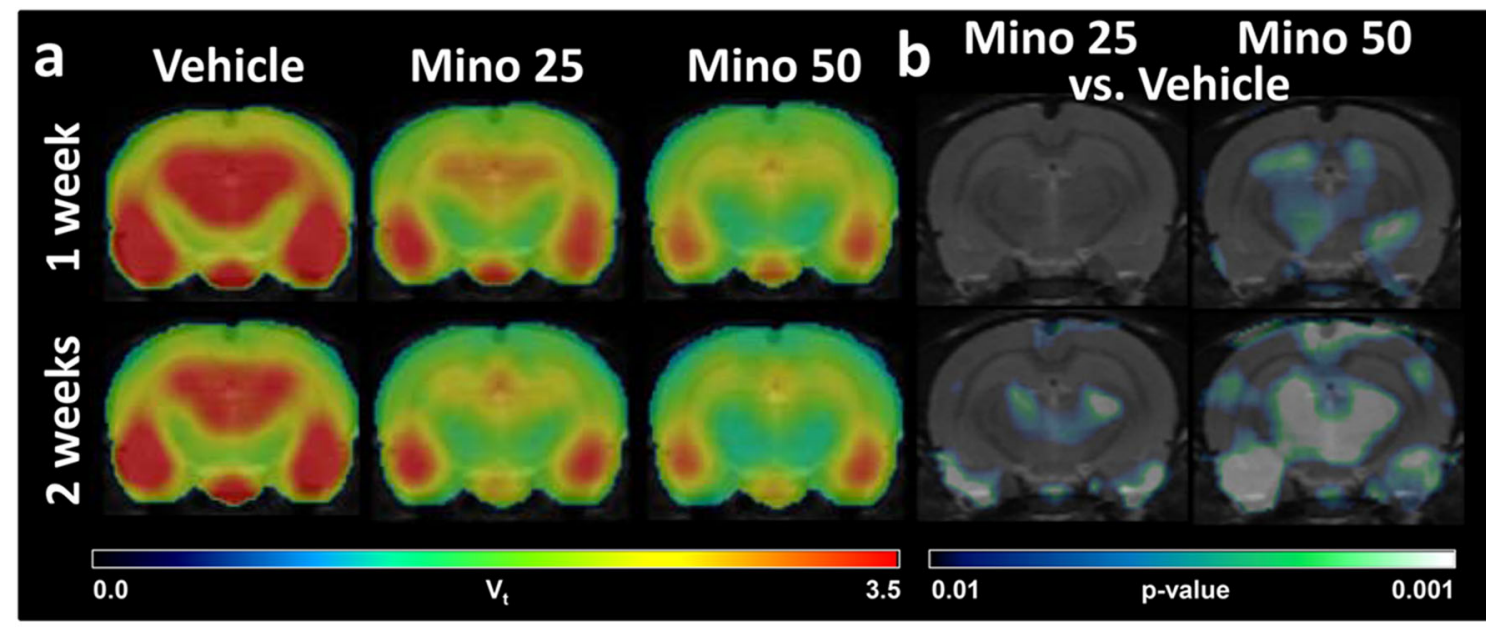

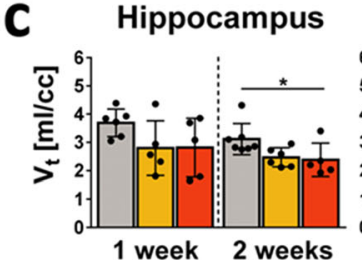
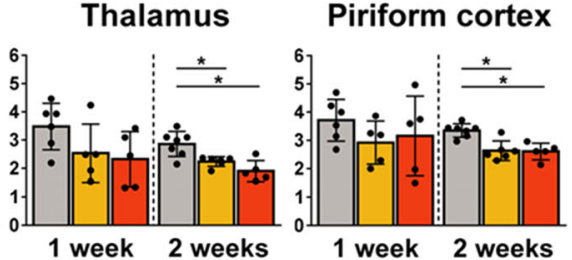

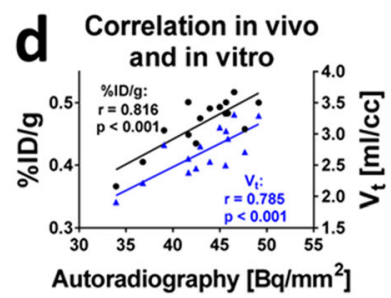

Fig. 1 Analysis of $\left[{ }^{18} \mathrm{~F}\right] \mathrm{GE} 180$ in vivo brain PET and in vitro autoradiography data in vehicle- and minocycline-treated rats after pilocarpine-induced status epilepticus (SE). (a) Averaged coronal $\left[{ }^{18} \mathrm{~F}\right] \mathrm{GE} 180$ volume of distribution $\left(\mathrm{V}_{\mathrm{t}}\right)$ maps $[\mathrm{ml} / \mathrm{cc}]$ at the level of maximal TSPO PET signal ( $3.6 \mathrm{~mm}$ caudal to bregma) 1 and 2 weeks after SE in vehicle- and minocycline-treated rats $(25 \mathrm{mg} / \mathrm{kg}$ or $50 \mathrm{mg} / \mathrm{kg}$ for 7 days). (b) Corresponding statistical parametric mapping (SPM) analysis comparing minocycline-treated versus vehicle-treated rats post-

this increase was less distinct but still statistically significant [17]. Minocycline treatment did not alter $\left[{ }^{18} \mathrm{~F}\right] \mathrm{GE} 180$ uptake compared to vehicle treatment in the analyzed VOIs at any of the time points studied (Fig. 3a, b). This was in line with the results of the SPM analysis which did not reveal significantly different voxels (Fig. 3a) and autoradiography (data not shown). In vivo data showed a strong correlation with in vitro autoradiography ( $r=0.695, p<0.001$, Fig. 3c).
$\mathrm{SE}$ ( $t$ test, $p<0.01$, minimum cluster size of 100 voxels). (c) Atlas-based analysis of $\left[{ }^{18} \mathrm{~F}\right] \mathrm{GE} 180 \mathrm{~V}_{\mathrm{t}}$ in epileptogenesis-associated brain regions. Data are mean \pm SD. Significant group differences calculated by oneway ANOVA and Dunnett's post hoc test are indicated by asterisks $(p<0.05)$. (d) Pearson correlation analysis of $\left[{ }^{18} \mathrm{~F}\right] \mathrm{GE} 180$ in vitro binding $\left[\mathrm{Bq} / \mathrm{mm}^{2}\right]$ to in vivo uptake $[\% \mathrm{ID} / \mathrm{g}]$ (indicated in black) and $\mathrm{V}_{\mathrm{t}}$ $[\mathrm{ml} / \mathrm{cc}]$ (indicated in blue). Mino, minocycline

Astrocyte activation, neurodegeneration, and neuronal cell dispersion was assessed in brain slices from mice 15 days after surgery (Fig. 4). Ipsilateral, vehicle-treated mice showed high score values for all parameters in all investigated brain areas. No impact of minocycline on neurodegeneration or astrocyte activation was observed (Fig. 4b, e). Nevertheless, neuronal cell dispersion in the dentate gyrus was reduced in minocycline-treated mice ( $p=0.040$; Fig. $4 c)$.
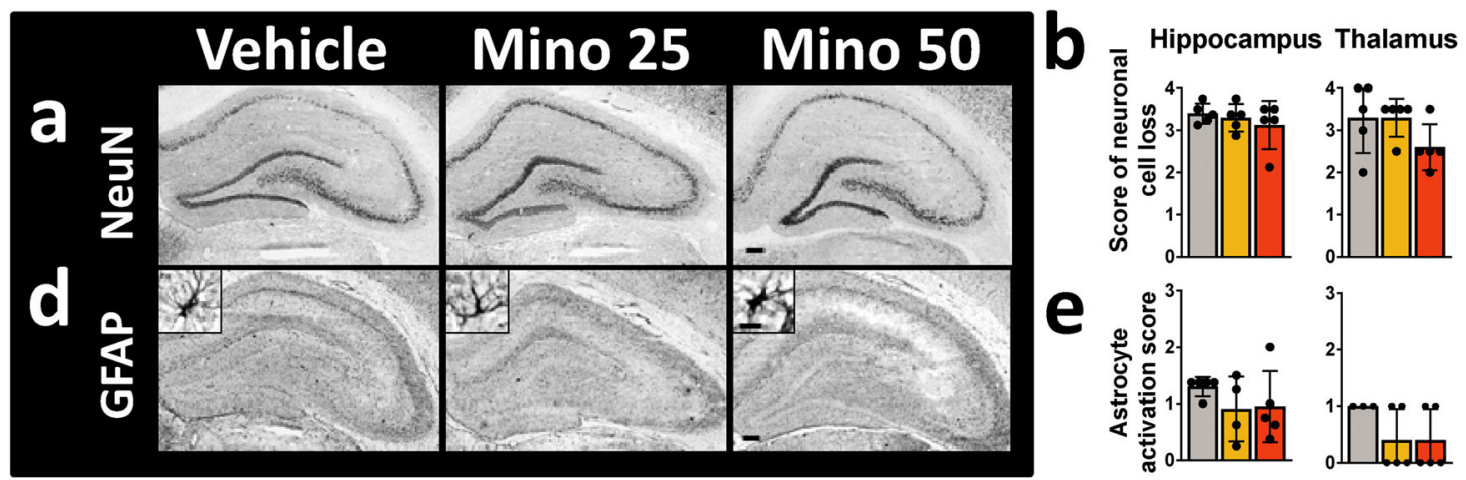

C Dentate hilus

Fig. 2 Histological analysis of neurodegeneration and astroglial activation in brain slices of vehicle- and minocycline-treated rats 2 weeks after pilocarpine-induced status epilepticus. Representative images of (a) neuronal nuclear antigen (NeuN)- or (d) glia acidic fibrillary protein (GFAP)-immunostained brain slices (scale bars:
$500 \mu \mathrm{m}$ for overview and $10 \mu \mathrm{m}$ for detailed images). Scoring of (b) neuronal cell loss and (e) astrocyte activation in selected brain regions. (c) Density of hippocampal hilus neurons per $\mathrm{mm}^{2}$. All data are mean \pm SD. Mino, minocycline 
Fig. 3 Analysis of TSPO brain PET in vehicle- and minocyclinetreated mice after status epilepticus (SE) induced by intrahippocampal kainate injection in mice. (a) Averaged $\left[{ }^{18} \mathrm{~F}\right] \mathrm{GE} 180$ uptake images [\% ID/ $\mathrm{g}]$ of vehicle- and minocyclinetreated mice $(50 \mathrm{mg} / \mathrm{kg}$ for 5 days) 1 and 2 weeks after SE at the level of maximal TSPO PET signal (2.92 $\mathrm{mm}$ caudal to bregma). Bottom row: Statistical parametric mapping (SPM) analysis comparing minocyclineand vehicle-treated animals ( $t$ test, $p<0.01$, minimum cluster size of 50 voxels). (b) Atlas-based analysis of $\left[{ }^{18} \mathrm{~F}\right] \mathrm{GE} 180$ uptake in selected brain areas. Data are mean \pm SD. (c) Pearson correlation analysis of $\left[{ }^{18} \mathrm{~F}\right] \mathrm{GE} 180$ in vitro binding $[\mathrm{Bq} /$ $\left.\mathrm{mm}^{2}\right]$ to in vivo uptake [\% ID/g].

Mino, minocycline

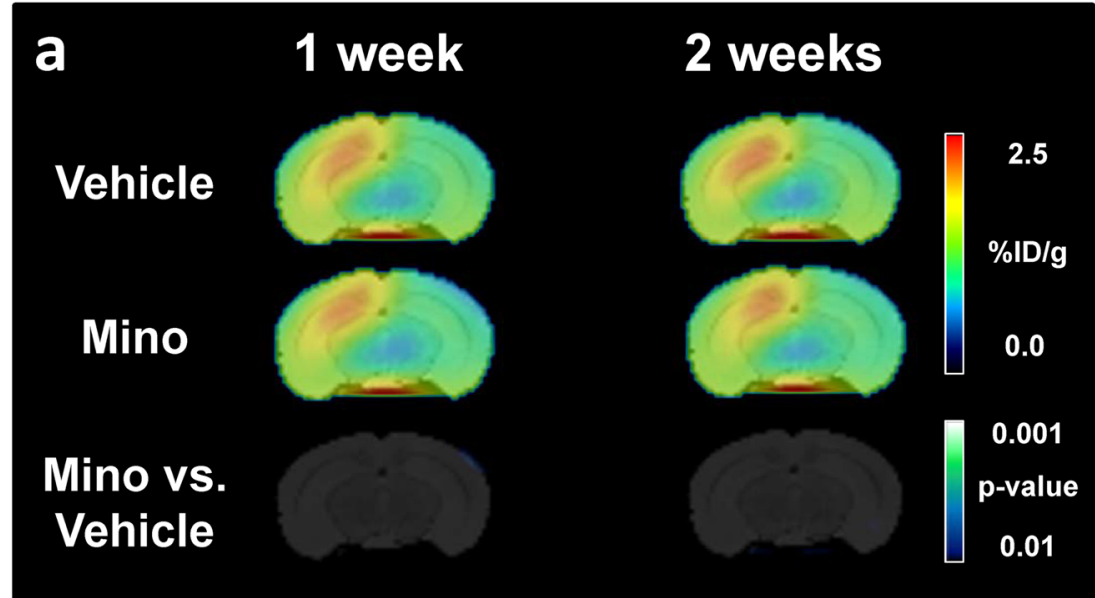

b

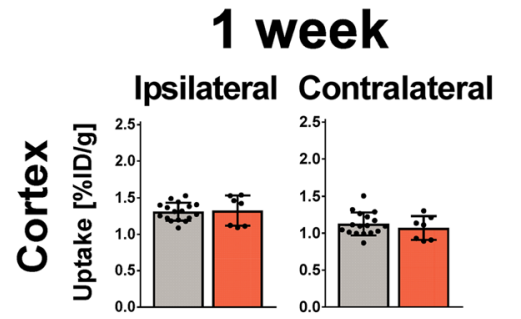

2 weeks
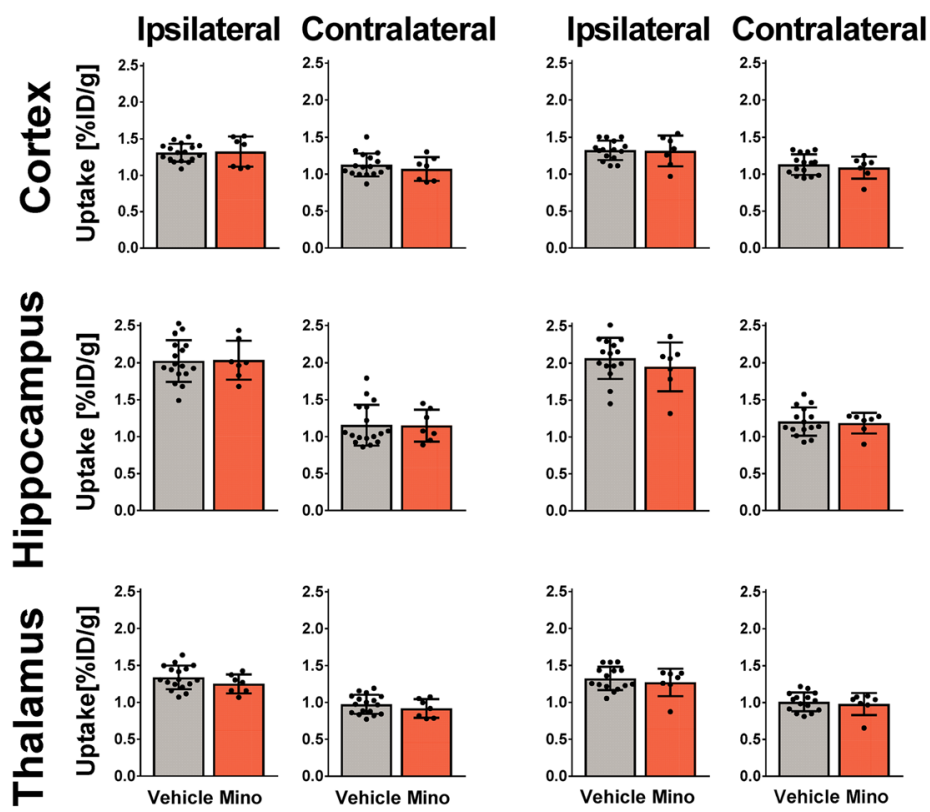

\section{Correlation in vivo}

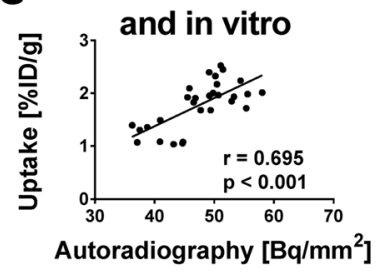

\section{Discussion}

In this study, we demonstrate by molecular in vivo imaging that minocycline treatment significantly reduces TSPO expression after pilocarpine-induced SE in rats, although it has no TSPO-reducing effect in the intrahippocampal kainate mouse model.

TSPO is primarily expressed on activated microglia and therefore allows in vivo evaluation of ongoing inflammatory processes [16]. Although TSPO might be expressed also in other brain cell types like astrocytes under certain conditions, there is no evidence that this is significantly the case during early epileptogenesis [33]. In PET studies, we have shown before that TSPO expression is substantially enhanced in epileptogenesisassociated brain regions within the first 2 weeks after SE in both applied animal models, which guided imaging time points and treatment duration for the present study [17, 18].

Minocycline, originally known for its anti-microbial mode of action, has been shown to attenuate the immune response via various pathways [20]. It reduces the production of pro- 
Fig. 4 Histologic analysis of neurodegeneration and astroglial activation in in brain slices of vehicle- and minocycline-treated mice 2 weeks after status epilepticus induction by intrahippocampal kainate injection. Representative images of (a) neuronal nuclear antigen (NeuN)- or (d) glia acidic fibrillary protein (GFAP)immunostained brain slices (scale bars: $500 \mu \mathrm{m}$ for overview and $10 \mu \mathrm{m}$ for detailed images). Scoring of (b) neuronal cell loss, (c) neuronal cell dispersion, or (e) astrocyte activation in selected brain regions. Data are mean \pm SD. Significant group difference calculated by Mann-Whitney test is indicated by asterisk $(p<0.05)$

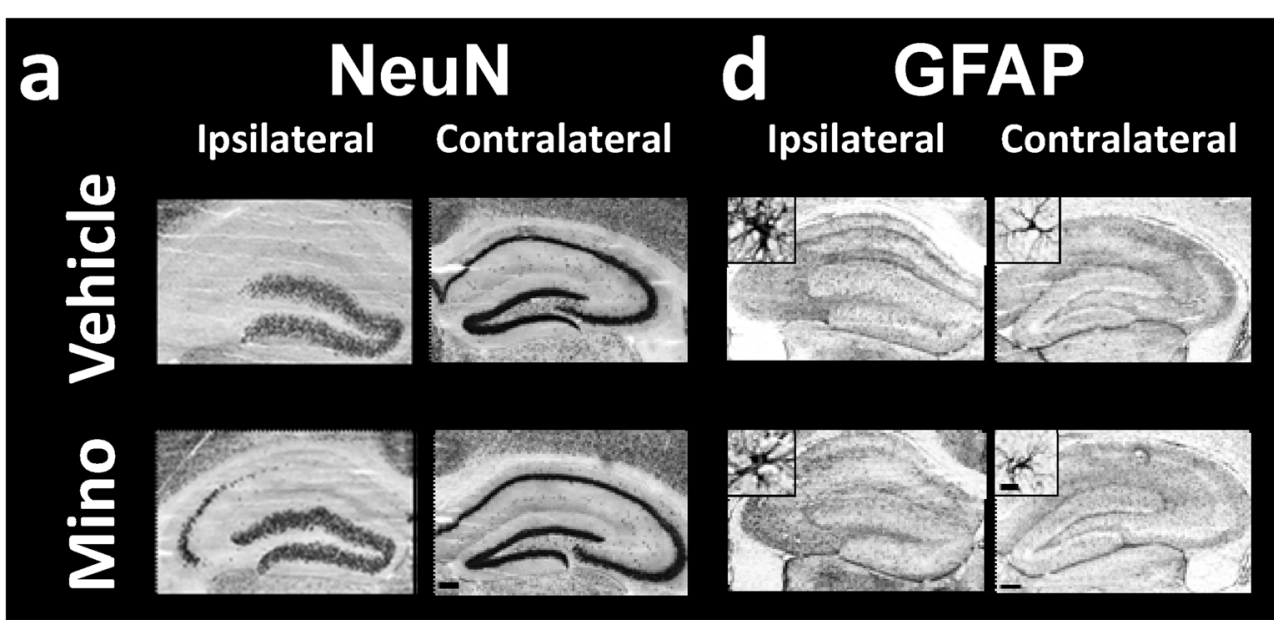

b

Ipsilateral Contralateral

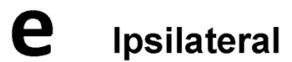

Contralateral
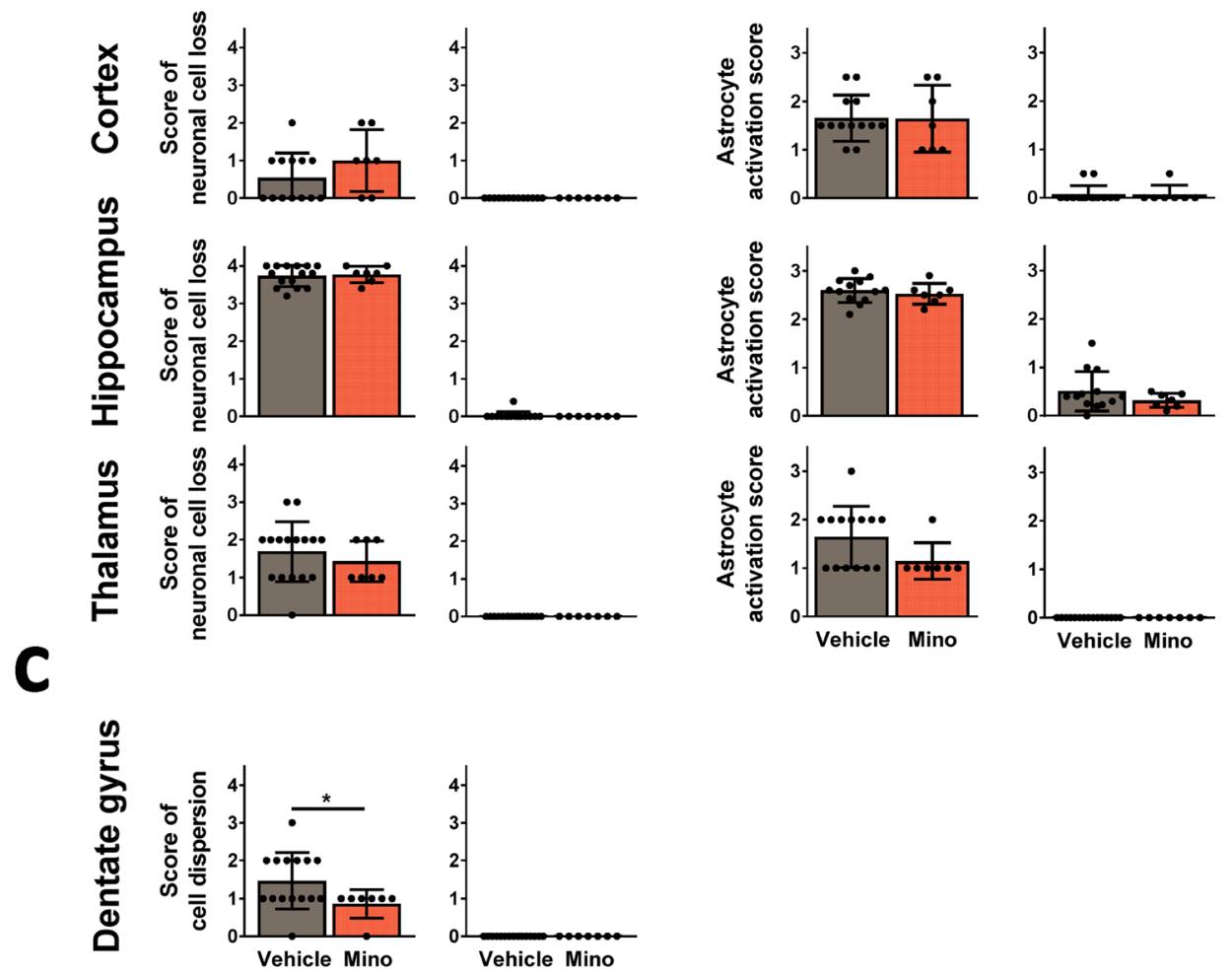

inflammatory cytokines and matrix metalloproteinases [21] and in an amyotrophic lateral sclerosis mouse model, it inhibited microglia to develop a more pro-inflammatory phenotype [43]. Furthermore, an inhibiting effect of minocycline on astrocytes, oligodendrocytes, neurons, and T cells has been reported $[44,45]$. Also a number of studies in rat and mouse models of epileptogenesis revealed effects both on neuroinflammation and the development of seizure susceptibility. In a two-hit SE mouse model, minocycline treatment after the first SE early in life revoked the increased susceptibility to the second SE later in life and reduced microglia activation [22]. Mice subjected to intrahippocampal kainate injection and

minocycline treatment showed decreased neurodegeneration [23]. Minocycline has also been shown to delay kindling acquisition in amygdala-kindled rats using a dose of $25 \mathrm{mg} / \mathrm{kg}$ [24]. Moreover, prolonged minocycline treatment following pilocarpine-induced SE in rats inhibited microglial activation assessed by immunohistochemistry during epileptogenesis, and reduced the frequency, duration, and severity of spontaneous recurrent seizures in the chronic phase of epilepsy [25].

Thus, the observed reduction of $\left[{ }^{18} \mathrm{~F}\right] \mathrm{GE} 180$ signal in the pilocarpine rat model generally lines up with prior published effects of minocycline. Nevertheless, in the study of Wang et al. [25], a dose of $45 \mathrm{mg} / \mathrm{kg}$ minocycline administered daily for 
14 days, beginning immediately after SE, inhibited microglial activation almost completely and exerted a disease-modifying effect. In the present study, the reduction of microglia activation was less intense and no attenuation of neurodegeneration or astrocyte activation was observed which may be due to the later start and shorter duration of the treatment. Although group size was based on power analysis, it seems that the actual data variation was bigger than estimated. Therefore, increasing the sample size might have resulted in significances, e.g., for TSPO signal reduction 1 week after SE (Fig. 1c). Minocycline has certain anti-convulsive effects which have been shown in animals as well as in a human case study [46, 47]. In line with this idea, a single minocycline injection in rats $(25 \mathrm{mg} / \mathrm{kg})$ given shortly after interruption of pilocarpine-induced SE found a certain degree of hippocampal neuroprotection [48]. Thus, SE might have been attenuated in the study by Wang et al. [25] as a consequence of direct minocycline administration causing less severe inflammation from the beginning. We chose a treatment start of $24 \mathrm{~h}$ after SE both to eliminate anti-convulsive and insult-modifying effects of minocycline. In addition, complete suppression of SE-induced neuroinflammatory response might also have harmful consequences [49]. In this regard, direct antiinflammatory corticosteroid treatment after interruption of pilocarpine-induced SE in rats has shown deteriorating rather than beneficial effects before [50]. Interestingly, a recent study by Russmann et al. [51] found only very limited minocycline effects on microglia activation or neurodegeneration using a different dosing scheme over 2 weeks after electrically induced $\mathrm{SE}$ in rats. Nevertheless, in this study, minocycline treatment had a positive impact on spatial learning, hyperactivity, and hyperlocomotion, whereas seizure development was not attenuated. Further, a study by Kwon et al. found no neuroprotective action when treating young rats with minocycline immediately after a double-hit insult (pilocarpine-mediated SE and lipopolysacharide injection) [52].

As the $50 \mathrm{mg} / \mathrm{kg}$ dose was more efficient than $25 \mathrm{mg} / \mathrm{kg}$ in rats after pilocarpine-induced SE, it was transferred to the mouse model. However, in the intrahippocampal kainate mouse model, TSPO PET did not detect any treatmentrelated changes. This is very unlikely be caused by a too low dose. First, applying a higher dose caused severe side effects, causing the termination of this experimental group. Second, a dose of $20 \mathrm{mg} / \mathrm{kg}$ minocycline injected once daily over 7 days in a mouse model of SE induced by systemic kainate administration during early life, decreased microglial activation to a pronounced extent [22]. This discrepancy to our findings may be attributed to differences in animal age or kainate administration route. It is also possible that in this context, the sensitivity of TSPO PET is not sufficient to reveal reduced neuroinflammation. However, minocycline treatment after focal cerebral ischemia in rats reduced uptake of the TSPO tracer $\left[{ }^{18} \mathrm{~F}\right] \mathrm{DPA}-714$ in the infarcted area [53]. In addition, in two other studies in the intrahippocampal kainate mouse model, we detected a reduced TSPO PET signal after anti-inflammatory treatment with curcumin or fingolimod 2 weeks after SE (unpublished data), confirming the general suitability of the chosen approach. In line with the observation that minocycline impacted epileptogenesis-related changes in rats, although this was not the case in mice, a study in neonatal rats and mice subjected to hypoxic-ischemic encephalopathy reported similar findings. Minocycline attenuated brain injury only in rats but even augmented it in mice, which may point towards a species-dependent addressability to minocycline [54]. Morphological evaluation of astrocyte activation did not reveal significant treatment effects. It has to be considered, however, that sole morphological analysis might not account for all anti-inflammatory effects on astrocytes.

Analysis of $\mathrm{V}_{\mathrm{t}}$ revealed more pronounced treatment effects than analysis of $\left[{ }^{18} \mathrm{~F}\right] \mathrm{GE} 180$ uptake in rats. These results need to be considered because for mice only uptake analysis was performed. Due to the repetitive PET scanning ruling out blood-sampling, the lack of a sufficient cerebral reference region and the small size of blood vessels combined with spill in from the surrounding hampering the generation of imagederived input functions, we could not establish a way to analyze $\left[{ }^{18} \mathrm{~F}\right] \mathrm{GE} 180$ kinetics in mice. The observation that $\mathrm{V}_{\mathrm{t}}$ can be more sensitive than uptake analysis was made before and can be explained by the fact that tracer uptake is influenced by various potential confounders such as tissue perfusion or metabolism, whereas kinetic analysis accounts for these [36]. This is also generally in line with a TSPO PET imaging study in TLE patients showing more pronounced $\mathrm{V}_{\mathrm{t}}$ differences, but also higher variation, compared to standardized uptake values [55]. Nevertheless, in line with earlier investigations [17, 18], the strong correlation of in vivo tracer uptake with the in vitro autoradiography data shown here for data from both rats and mice supports the application of uptake analyses.

Although the focus of this TSPO PET study was assessing early anti-inflammatory effects of minocycline treatment, other studies performed a follow up of rats after SE to the chronic disease phase: Bertoglio et al. conclude from their findings that TSPO PET might serve as a biomarker for early prediction of epileptic seizure frequency [56], and a further study identified TSPO PET as a predictor of treatment response to anti-seizure drugs [57]. These findings support the idea that TSPO PET might also be suitable for early prediction of response to anti-epileptogenic or disease-modifying therapies; however, this remains to be assessed in future studies. In the present study TSPO PET identified minocycline-mediated reduction of neuroinflammation during epileptogenesis in the lithium-pilocarpine rat model, but not in the intrahippocampal kainate mouse model of epileptogenesis. It has to be considered that minocycline's anti-epileptogenic properties might be mediated by further mechanisms beyond a reduction of microglia activation. Nonetheless, the described setup can now be used to preclinically investigate individual treatment 
responses during the latent phase and their correlation to chronic disease outcome. Furthermore, PET may be utilized to identify purposeful drug doses and treatment durations. TSPO PET might also be a valuable tool for evaluation of non-anti-inflammatory treatments preventing secondary neuroinflammation (and therefore increased TSPO expression). In addition, tracers and imaging protocols are very similar for patients, facilitating translation of findings to the clinic for future personalized treatment approaches.

Acknowledgments Open access funding provided by Projekt DEAL. The authors thank the preclinical molecular imaging and the radiochemistry staff of the Department of Nuclear Medicine for their support. This study was funded by the European Seventh's Framework Program (FP7/ 2007-2013) under grant agreement no. 602102 (EPITARGET). MBr and IL were supported by a scholarship from the Konrad-Adenauer Stiftung. BJW was supported by a scholarship from the Studienstiftung des Deutschen Volkes. We thank GE Healthcare for providing the general method and access to the precursor for $\left[{ }^{18} \mathrm{~F}\right] \mathrm{GE} 180$ synthesis.

Required Author Forms Disclosure forms provided by the authors are available with the online version of this article.

\section{Compliance with Ethical Standards}

Conflict of Interest The authors declare that they have no conflict of interest.

Ethical Publication Statement We confirm that we have read the Journal's position on issues involved in ethical publication and affirm that this report is consistent with those guidelines.

Open Access This article is licensed under a Creative Commons Attribution 4.0 International License, which permits use, sharing, adaptation, distribution and reproduction in any medium or format, as long as you give appropriate credit to the original author(s) and the source, provide a link to the Creative Commons licence, and indicate if changes were made. The images or other third party material in this article are included in the article's Creative Commons licence, unless indicated otherwise in a credit line to the material. If material is not included in the article's Creative Commons licence and your intended use is not permitted by statutory regulation or exceeds the permitted use, you will need to obtain permission directly from the copyright holder. To view a copy of this licence, visit http://creativecommons.org/licenses/by/4.0/.

\section{References}

1. WHO. Fact sheets - Epilepsy. [online] 2019 [cited 2019 August 12]; Available from: https://www.who.int/news-room/fact-sheets/detail/ epilepsy.

2. Löscher W. The holy grail of epilepsy prevention: Preclinical approaches to antiepileptogenic treatments. Neuropharmacology. 2019 Apr 11

3. Pitkänen A, Lukasiuk K, Dudek FE, Staley KJ. Epileptogenesis. Cold Spring Harb Perspect Med. 2015 Sep 18;5(10).

4. Spencer SS. Neural networks in human epilepsy: evidence of and implications for treatment. Epilepsia. 2002 Mar;43(3):219-27.

5. Löscher W, Brandt C. Prevention or modification of epileptogenesis after brain insults: Experimental approaches and translational research. Pharmacol Rev. 2010;62(4):668-700.
6. Pitkänen A, Lukasiuk K. Molecular and cellular basis of epileptogenesis in symptomatic epilepsy. Epilepsy Behav. 2009 Jan;14:16-25.

7. Stables JP, Bertram E, Dudek FE, Holmes G, Mathern G, Pitkänen A, et al. Therapy discovery for pharmacoresistant epilepsy and for disease-modifying therapeutics: summary of the NIH/NINDS/AES models II workshop. Epilepsia. 2003 12;44(12):1472-8.

8. Löscher W, Hirsch LJ, Schmidt D. The enigma of the latent period in the development of symptomatic acquired epilepsy - Traditional view versus new concepts. Epilepsy Behav. 2015 Nov;52(Pt A): 78-92.

9. Vezzani A, Friedman A, Dingledine RJ. The role of inflammation in epileptogenesis. Neuropharmacology. 2013 Jun;69:16-24.

10. Devinsky O, Vezzani A, Najjar S, De Lanerolle NC, Rogawski MA. Glia and epilepsy: excitability and inflammation. Trends Neurosci. 2013;36(3):174-84.

11. Amhaoul H, Hamaide J, Bertoglio D, Reichel SN, Verhaeghe J, Geerts E, et al. Brain inflammation in a chronic epilepsy model: Evolving pattern of the translocator protein during epileptogenesis. Neurobiol Dis. 2015 2015-Oct;82:526-39.

12. Ravizza T, Gagliardi B, Noe F, Boer K, Aronica E, Vezzani A. Innate and adaptive immunity during epileptogenesis and spontaneous seizures: Evidence from experimental models and human temporal lobe epilepsy. Neurobiol Dis. 2008 Jan;29(1):142-60.

13. Pernot F, Heinrich C, Barbier L, Peinnequin A, Carpentier P, Dhote $\mathrm{F}$, et al. Inflammatory changes during epileptogenesis and spontaneous seizures in a mouse model of mesiotemporal lobe epilepsy. Epilepsia. 2011 Dec;52(12):2315-25.

14. Vezzani A, Balosso S, Ravizza T. Neuroinflammatory pathways as treatment targets and biomarkers in epilepsy. Nature Reviews Neurology. 2019 2019/08/01;15(8):459-72.

15. Banati RB. Visualising microglial activation in vivo. Glia. 2002;40(2):206-17.

16. Dupont AC, Largeau B, Santiago Ribeiro MJ, Guilloteau D, Tronel C, Arlicot N. Translocator protein-18 kDa (TSPO) positron emission tomography (PET) imaging and its clinical impact in neurodegenerative diseases. Int J Mol Sci. 2017 Apr 7;18(4).

17. Brackhan M, Bascunana P, Ross TL, Bengel FM, Bankstahl JP, Bankstahl M. [(18) F]GE180 positron emission tomographic imaging indicates a potential double-hit insult in the intrahippocampal kainate mouse model of temporal lobe epilepsy. Epilepsia. 2018 Jan 24;59(3):617-26.

18. Brackhan M, Bascunana P, Postema JM, Ross TL, Bengel FM, Bankstahl M, et al. Serial quantitative TSPO-targeted PET reveals peak microglial activation up to 2 weeks after an epileptogenic brain insult. J Nucl Med. 2016 Aug;57(8):1302-8.

19. Ravizza T, Vezzani A. Pharmacological targeting of brain inflammation in epilepsy: Therapeutic perspectives from experimental and clinical studies. Epilepsia Open. 2018;3(S2):133-42.

20. Möller T, Bard F, Bhattacharya A, Biber K, Campbell B, Dale E, et al. Critical data-based re-evaluation of minocycline as a putative specific microglia inhibitor. Glia. 2016;64(10):1788-94.

21. Bialer M, Johannessen SI, Levy RH, Perucca E, Tomson T, White HS. Progress report on new antiepileptic drugs: A summary of the thirteenth Eilat conference on new antiepileptic drugs and devices (EILAT XIII). Epilepsia. 2017 Feb;58(2):181-221.

22. Abraham J, Fox PD, Condello C, Bartolini A, Koh S. Minocycline attenuates microglia activation and blocks the long-term epileptogenic effects of early-life seizures. Neurobiol Dis. 2012 May;46(2): 425-30.

23. Heo K, Cho YJ, Cho KJ, Kim HW, Kim HJ, Shin HY, et al. Minocycline inhibits caspase-dependent and -independent cell death pathways and is neuroprotective against hippocampal damage after treatment with kainic acid in mice. Neurosci Lett. 2006 May 8;398(3):195-200. 
24. Beheshti Nasr SM, Moghimi A, Mohammad-Zadeh M, Shamsizadeh A, Noorbakhsh SM. The effect of minocycline on seizures induced by amygdala kindling in rats. Seizure. 2013 Oct;22(8):670-4.

25. Wang N, Mi X, Gao B, Gu J, Wang W, Zhang Y, et al. Minocycline inhibits brain inflammation and attenuates spontaneous recurrent seizures following pilocarpine-induced status epilepticus. Neuroscience. 2015 Feb;287:144-56.

26. Twele F, Tollner K, Brandt C, Loscher W. Significant effects of sex, strain, and anesthesia in the intrahippocampal kainate mouse model of mesial temporal lobe epilepsy. Epilepsy Behav. 2016 Feb;55:47-56.

27. Brandt C, Bankstahl M, Tollner K, Klee R, Loscher W. The pilocarpine model of temporal lobe epilepsy: Marked intrastrain differences in female Sprague-Dawley rats and the effect of estrous cycle. Epilepsy Behav. 2016 Aug;61:141-52.

28. Löscher W, Ferland RJ, Ferraro TN. The relevance of inter- and intrastrain differences in mice and rats and their implications for models of seizures and epilepsy. Epilepsy \& Behavior. 2017;73: 214-35.

29. Rattka M, Brandt C, Bankstahl M, Broer S, Loscher W. Enhanced susceptibility to the GABA antagonist pentylenetetrazole during the latent period following a pilocarpine-induced status epilepticus in rats. Neuropharmacology. 2011 Feb-Mar;60(2-3):505-12.

30. Racine RJ. Modification of seizure activity by electrical stimulation 2. motor seizure. Electroencephalogr Clin Neurophysiol. 1972;32(3):281-\&.

31. Mirrione MM, Schiffer WK, Fowler JS, Alexoff DL, Dewey SL, Tsirka SE. A novel approach for imaging brain-behavior relationships in mice reveals unexpected metabolic patterns during seizures in the absence of tissue plasminogen activator. Neuroimage. [Article]. 2007 Oct;38(1):34-42.

32. Schiffer WK, Mirrione MM, Biegon A, Alexoff DL, Patel V, Dewey SL. Serial microPET measures of the metabolic reaction to a microdialysis probe implant. J Neurosci Methods. 2006 Sep 15;155(2):272-84

33. Mirrione MM, Schiffer WK, Siddiq M, Dewey SL, Tsirka SE. PET imaging of glucose metabolism in a mouse model of temporal lobe epilepsy. Synapse. [Article]. 2006 Feb;59(2):119-21.

34. Schwarz AJ, Danckaert A, Reese T, Gozzi A, Paxinos G, Watson C, et al. A stereotaxic MRI template set for the rat brain with tissue class distribution maps and co-registered anatomical atlas: Application to pharmacological MRI. Neuroimage. 2006;32(2): 538-50.

35. Bascuñana P, Brackhan M, Leiter I, Keller H, Jahreis I, Ross TL, et al. Divergent metabolic substrate utilization in brain during epileptogenesis precedes chronic hypometabolism. J Cereb Blood Flow Metab. 2019;0(0):0271678X18809886.

36. Thackeray JT, Bankstahl JP, Bengel FM. Impact of image-derived input function and fit time intervals on patlak quantification of myocardial glucose uptake in mice. J Nucl Med. 2015 Oct;56(10):1615-21.

37. Jucaite A, Cselenyi Z, Arvidsson A, Ahlberg G, Julin P, Varnas K, et al. Kinetic analysis and test-retest variability of the radioligand $[11 \mathrm{C}](\mathrm{R})-\mathrm{PK} 11195$ binding to TSPO in the human brain - a PET study in control subjects. EJNMMI Res. 2012 Apr 23;2:15.

38. Paxinos G, Watson $\mathrm{C}$. The rat brain in stereotaxic coordinates. Sydney: Academic Press; 2007.

39. Paxinos G, Franklin KBJ. Paxinos and Franklin's The mouse brain in stereotaxic coordinates 2012.

40. Nguyen DL, Wimberley C, Truillet C, Jego B, Caille F, Pottier G, et al. Longitudinal positron emission tomography imaging of glial cell activation in a mouse model of mesial temporal lobe epilepsy: Toward identification of optimal treatment windows. Epilepsia. 2018 Jun;59(6):1234- 44.
41. Müller CJ, Bankstahl M, Gröticke I, Löscher W. Pilocarpine vs. lithium-pilocarpine for induction of status epilepticus in mice: development of spontaneous seizures, behavioral alterations and neuronal damage. Eur J Pharmacol. 2009 Oct 1;619(1-3):15-24.

42. Polascheck N, Bankstahl M, Löscher W. The COX-2 inhibitor parecoxib is neuroprotective but not antiepileptogenic in the pilocarpine model of temporal lobe epilepsy. Exp Neurol. 2010;224(1): 219-33.

43. Kobayashi K, Imagama S, Ohgomori T, Hirano K, Uchimura K, Sakamoto K, et al. Minocycline selectively inhibits M1 polarization of microglia. Cell Death Dis. 2013 Mar 7;4:e525.

44. Garrido-Mesa N, Zarzuelo A, Galvez J. Minocycline: far beyond an antibiotic. Br J Pharmacol. 2013 May;169(2):337-52.

45. Hou Y, Xie G, Liu X, Li G, Jia C, Xu J, et al. Minocycline protects against lipopolysaccharide-induced cognitive impairment in mice. Psychopharmacology (Berl). 2016 Mar;233(5):905-16.

46. Wang DD, Englot DJ, Garcia PA, Lawton MT, Young WL. Minocycline- and tetracycline-class antibiotics are protective against partial seizures in vivo. Epilepsy Behav. 2012 Jul;24(3): 314-8.

47. Nowak M, Strzelczyk A, Reif PS, Schorlemmer K, Bauer S, Norwood BA, et al. Minocycline as potent anticonvulsant in a patient with astrocytoma and drug resistant epilepsy. Seizure. 2012 Apr;21(3):227-8.

48. Arisi G, Foresti M, Montañez A, A Shapiro L. Minocycline ameliorates neuronal loss after pilocarpine-induced status epilepticus. J Neurol Disord Stroke. 2014;2:1055.

49. DiSabato DJ, Quan N, Godbout JP. Neuroinflammation: the devil is in the details. J Neurochem. 2016 Oct;139 Suppl 2:136-53.

50. Duffy BA, Chun KP, Ma D, Lythgoe MF, Scott RC. Dexamethasone exacerbates cerebral edema and brain injury following lithium-pilocarpine induced status epilepticus. Neurobiol Dis. 2014 Mar;63:229-36.

51. Russmann V, Goc J, Boes K, Ongerth T, Salvamoser JD, Siegl C, et al. Minocycline fails to exert antiepileptogenic effects in a rat status epilepticus model. Eur J Pharmacol. 2016 Jan 15;771:29-39.

52. Kwon YS, Pineda E, Auvin S, Shin D, Mazarati A, Sankar R. Neuroprotective and antiepileptogenic effects of combination of anti-inflammatory drugs in the immature brain. J Neuroinflammation. 2013 Feb 26;10:30.

53. Martin A, Boisgard R, Kassiou M, Dolle F, Tavitian B. Reduced $\mathrm{PBR} / \mathrm{TSPO}$ expression after minocycline treatment in a rat model of focal cerebral ischemia: a PET study using [(18)F]DPA-714. Mol Imaging Biol. $2011 \mathrm{Feb}$;13(1):10-5.

54. Tsuji M, Wilson MA, Lange MS, Johnston MV. Minocycline worsens hypoxic-ischemic brain injury in a neonatal mouse model. Exp Neurol. 2004 Sep;189(1):58-65.

55. Gershen LD, Zanotti-Fregonara P, Dustin IH, Liow JS, Hirvonen J, Kreisl WC, et al. Neuroinflammation in temporal lobe epilepsy measured using positron emission tomographic imaging of translocator protein. JAMA Neurol. 2015 Aug;72(8):882-8.

56. Bertoglio D, Verhaeghe J, Santermans E, Amhaoul H, Jonckers E, Wyffels L, et al. Non-invasive PET imaging of brain inflammation at disease onset predicts spontaneous recurrent seizures and reflects comorbidities. Brain Behavior and Immunity. 2017 Mar;61:69-79.

57. Bogdanovic RM, Syvänen S, Michler C, Russmann V, Eriksson J, Windhorst AD, et al. (R)- C-11 PK11195 brain uptake as a biomarker of inflammation and antiepileptic drug resistance: Evaluation in a rat epilepsy model. Neuropharmacology. 2014 Oct;85:104-12.

Publisher's Note Springer Nature remains neutral with regard to jurisdictional claims in published maps and institutional affiliations. 